\title{
Analysis of Key Technologies in the Design of Small and Medium Span Basket Type Steel Box Tied Arch Bridge
}

\author{
Zhongyu Wang* \\ China Merchants Chongqing Communications Technology Research \& Design Institute CO. LTD. Chongqing 400067, China \\ *Corresponding author: Zhongyu Wang, ccrdiyu@gmail.com

\begin{abstract}
In order to ensure the construction quality and safety of small and medium span basket type steel box tied arch bridge, this paper takes a practical project as an example to analyze the key technologies in its design process. It is hoped that this analysis can provide corresponding reference for the design and construction of this kind of arch bridge.
\end{abstract}

Keywords: Medium and small span; Basket type steel box tie arch bridge; Arch Bridge Design; Key technology

Publication date: July 2021; Online publication: July 31, 2021

\section{Introduction}

In the design process of medium and small span basket type steel box tied arch bridge, the most key technology is to analyze and check some important local positions, such as steel structure arch box lifting lug, lower anchor head of suspender, steel-concrete joint section and so on. In this way, the design parameters of each key part can be effectively ensured, so as to ensure the design quality of steel structure tied arch bridge and meet its actual construction and application needs ${ }^{[1]}$.

\subsection{Project overview}

This paper studies the local design checking calculation of a small and medium-sized span basket type steel box tied arch bridge across the river in Southwest China. The bridge project belongs to the main road of the city, the total length is $106.199 \mathrm{~m}$, the design speed is $40 \mathrm{~km}$, the road width is $27.2 \mathrm{~m}$ with two-way four lanes, the structure is the steel box arch bridge, the main span is $74 \mathrm{~m}$, the steel box girder material choice is Q345C steel, and the concrete system beam is C50 concrete. This paper mainly analyzes the local key technologies such as arch box lifting lug, anchor head under suspender and steel-concrete joint section.

\section{Analysis of key technologies in design}

\subsection{Local analysis of arch box lug position}

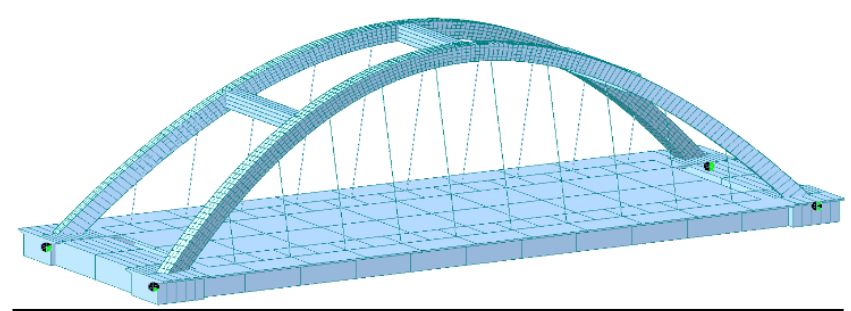

Figure 1. Calculation model of the main beam in this project

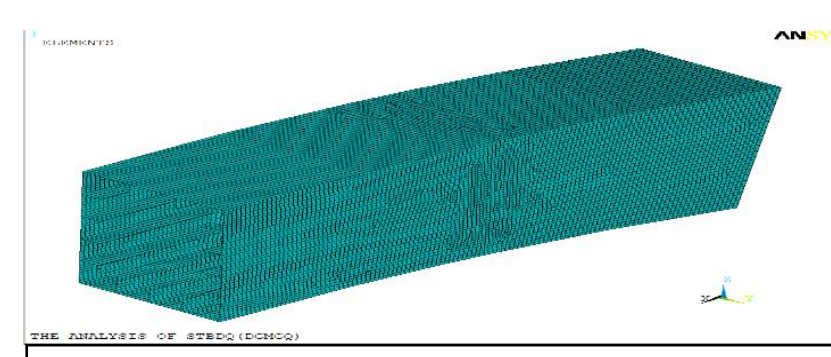

Figure 2. Finite element model of plate and shell in suspender anchorage zone 
According to the member system calculating analysis (Figure 1), the internal force of the suspender at the vault position is the largest. Under the combined envelope of the limit state of bearing capacity, the maximum internal force of the suspender is $2856 \mathrm{kN}$.

The box arch section of the vault area is selected for local analysis of the anchorage area of the derrick (Figure 2). ANSYS was used to establish the plate and shell finite element model of related segments, SHELL181 elastic-plastic element was used as element type, and the thickness of plate and shell element was given by real constant. The arc length method is used to analyze the ultimate bearing capacity.

Lifting point displacements and lifting point forces of each sub-step were extracted to form the load displacement curve. When the tangent stiffness of the load displacement curve approached 0 , it was the ultimate bearing capacity of the anchorage zone of the suspender. The following is the cloud diagram of equivalent stress in the anchorage zone of (step1) lifting point. (Figure 3)

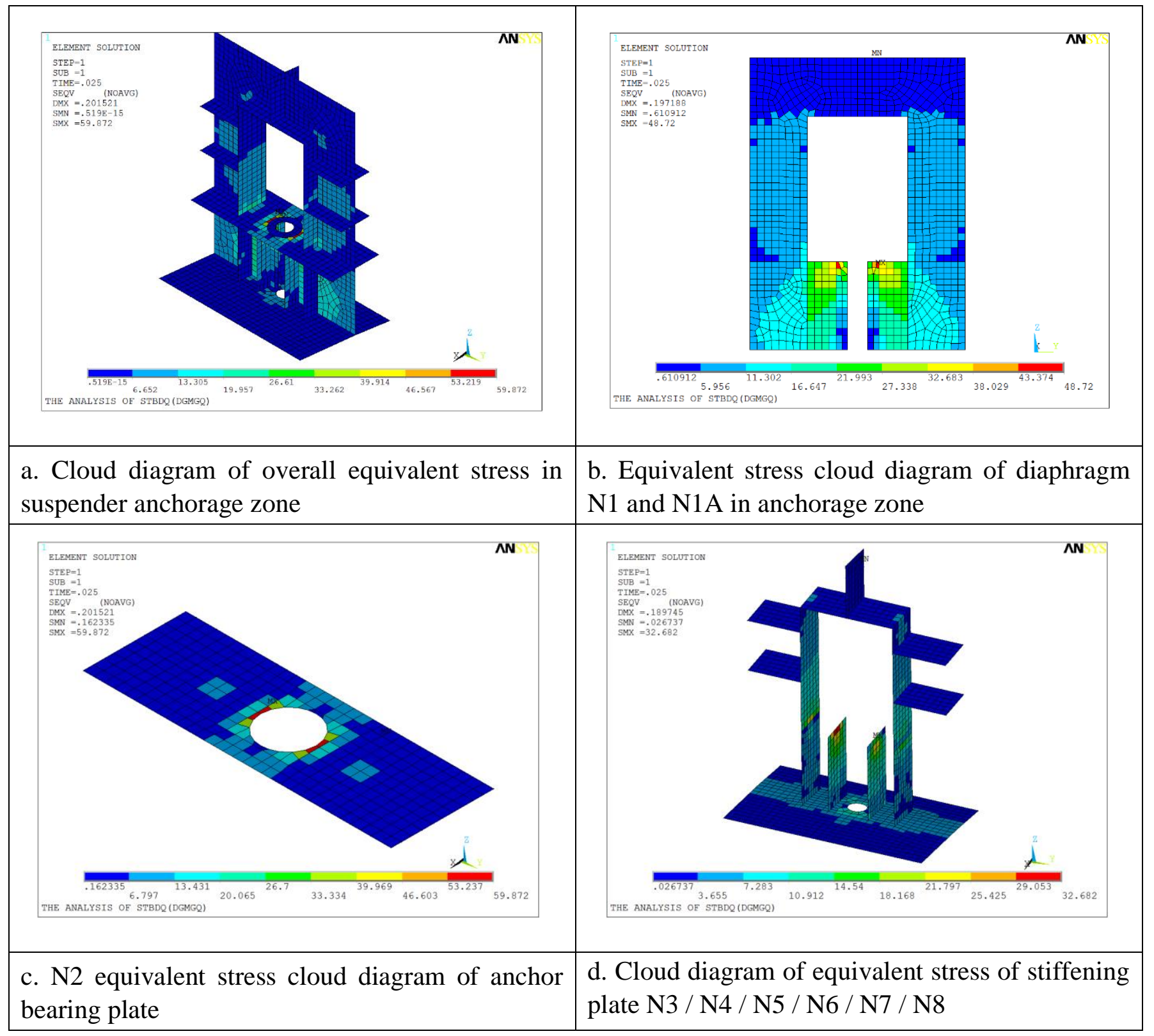

Figure 3. Cloud diagram of equivalent stress in suspender anchorage zone (step1) 
The equivalent stress diagram step1 14 and load displacement curve of suspender anchorage zone can also be obtained as in Table 1.

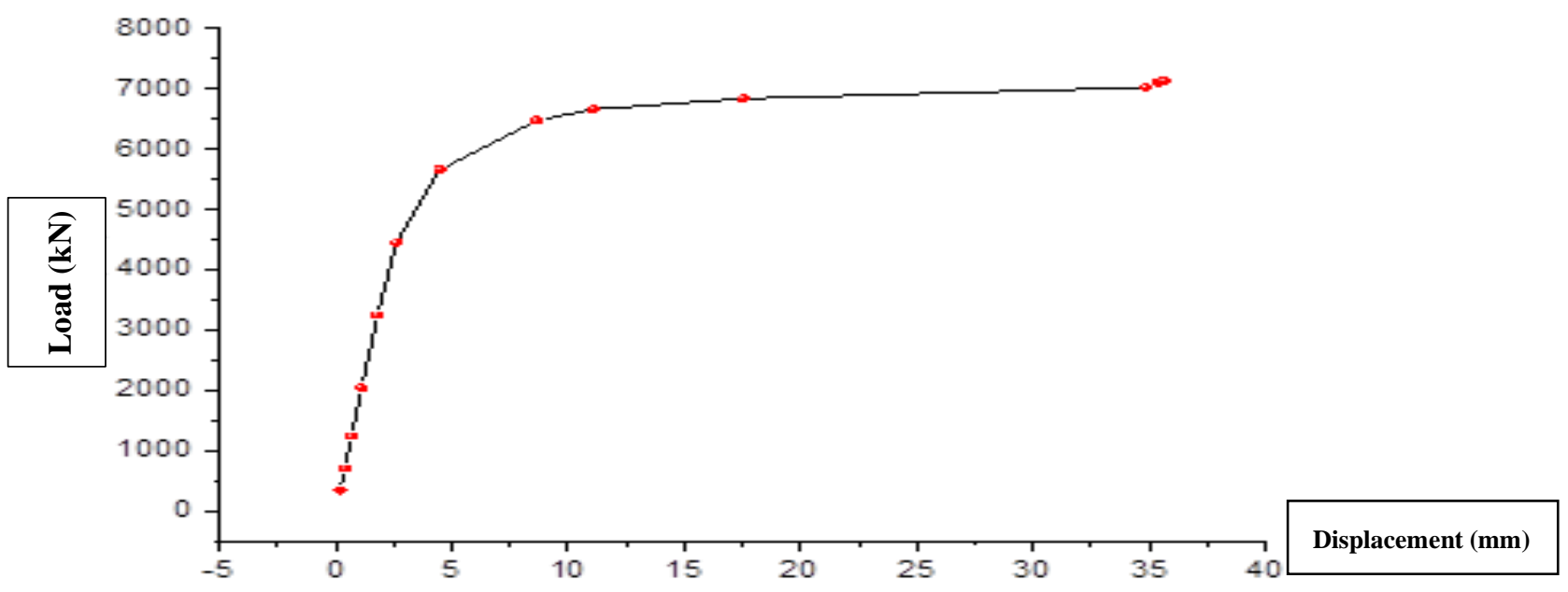

Figure 4. Load displacement curve of the suspender anchorage zone

Table 1. Load displacement table

\begin{tabular}{|c|c|c|}
\hline Step & Load $(\mathbf{k N})$ & Displacement (mm) \\
\hline 1 & 357 & 0.2 \\
\hline 2 & 714 & 0.4 \\
\hline 3 & 1250 & 0.7 \\
\hline 4 & 2053 & 1.1 \\
\hline 5 & 3258 & 1.8 \\
\hline 6 & 4463 & 2.6 \\
\hline 7 & 5667 & 4.5 \\
\hline 8 & 6481 & 8.6 \\
\hline 9 & 6663 & 11.1 \\
\hline 10 & 6845 & 17.5 \\
\hline 11 & 7027 & 34.9 \\
\hline 12 & 7109 & 35.4 \\
\hline 13 & 7126 & 35.5 \\
\hline 14 & 7142 & 35.6 \\
\hline
\end{tabular}

It can be seen from the load displacement curve (Figure 4) that when the tangent stiffness tends to 0 , the corresponding ultimate bearing capacity is $7027 \mathrm{kN}$, which is greater than the maximum suspender force of $2856 \mathrm{kN}$ and the safety factor is 2.46 . Meet the requirements.

\subsection{Local analysis of anchor head under suspender}

The local main girder of the lower anchor head of the suspender is selected for analysis (Figure 5). The model adopts the solid element, and the most unfavorable suspender force of the standard combination envelope is applied to the anchor bearing plate of the lower anchor head in the form of surface pressure load ${ }^{[2]}$. 


\section{Below are figures 5-10:}

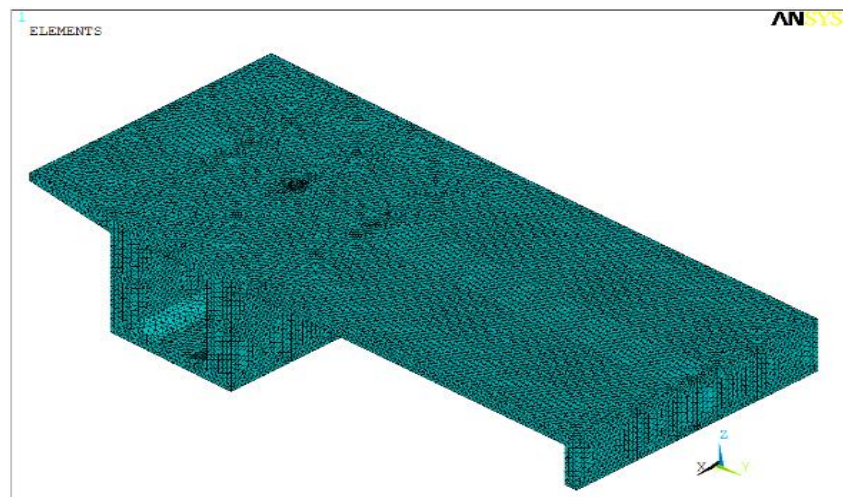

THE ANALYSIS OF STBDQ (DGXMT)

Figure 5. Finite element model for local analysis of lower anchor head of suspender

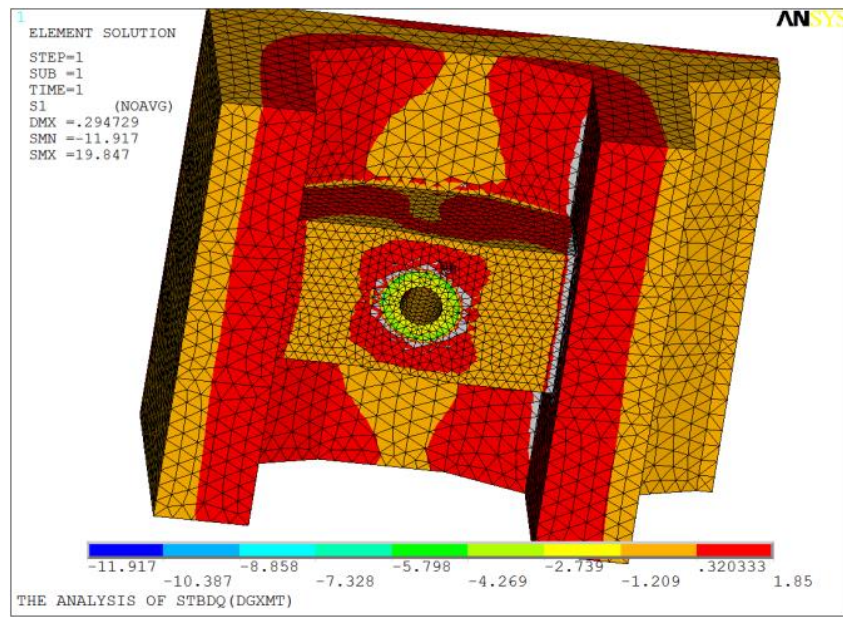

Figure 7. Cloud diagram of principal tensile stress of anchor head under suspender

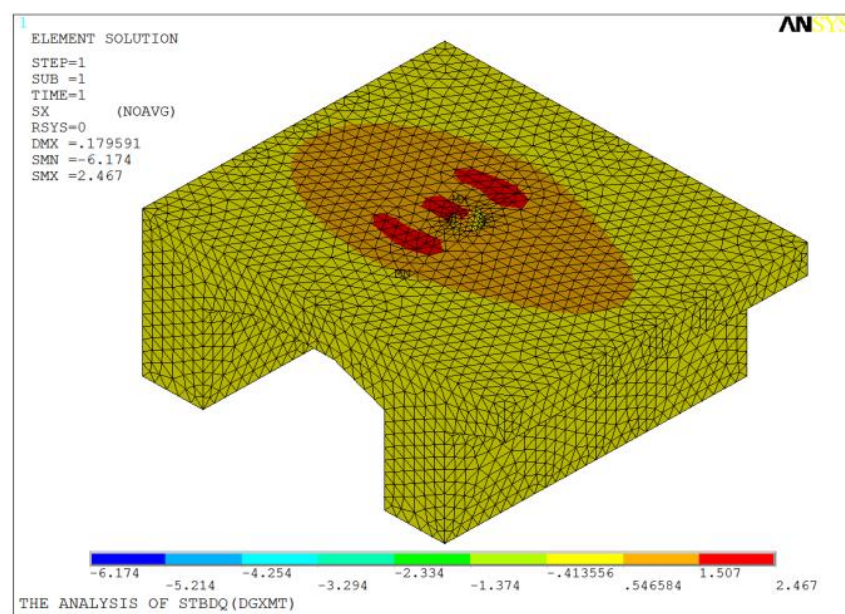

Figure 9. Normal stress of anchor head top plate under suspender along the bridge

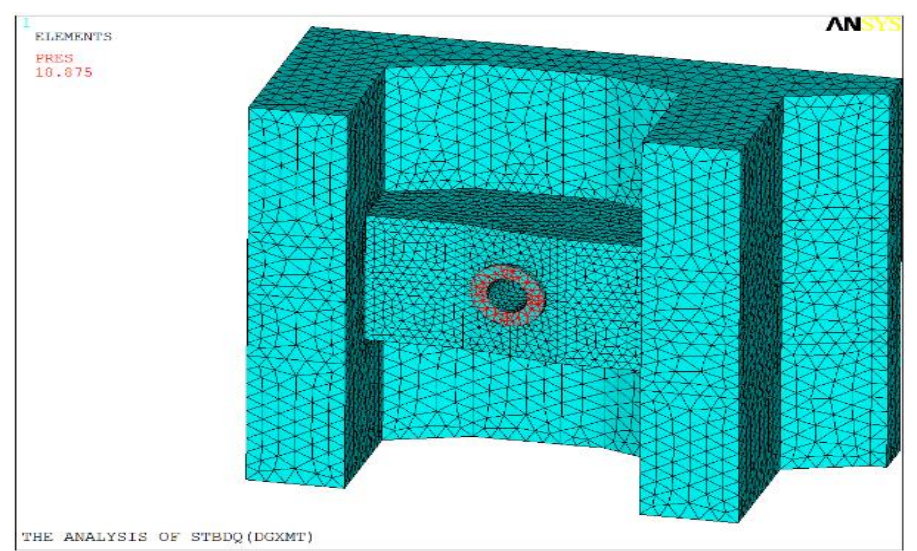

Figure 6. Enlarged finite element model of anchor head under suspender

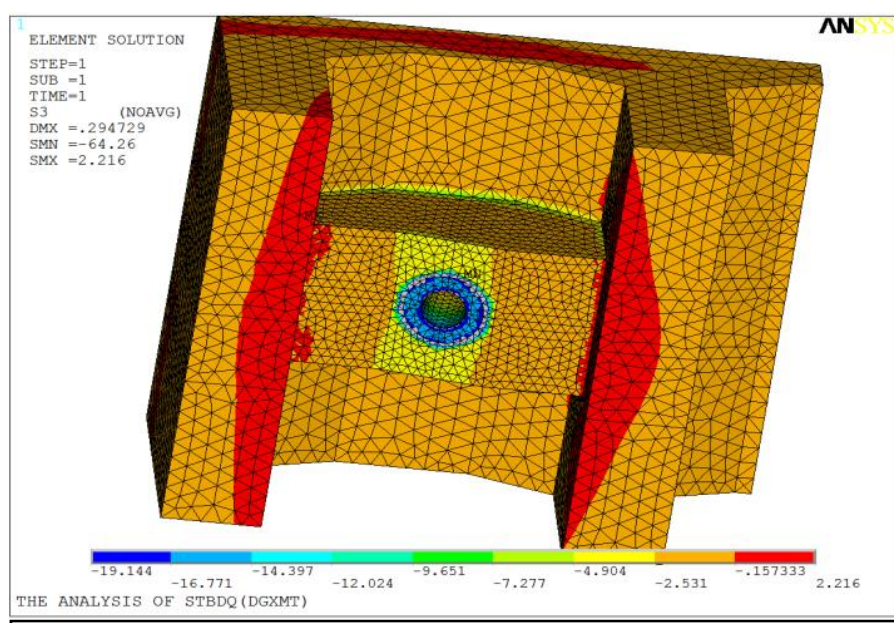

Figure 8. Cloud diagram of principal compressive stress of anchor head under suspender

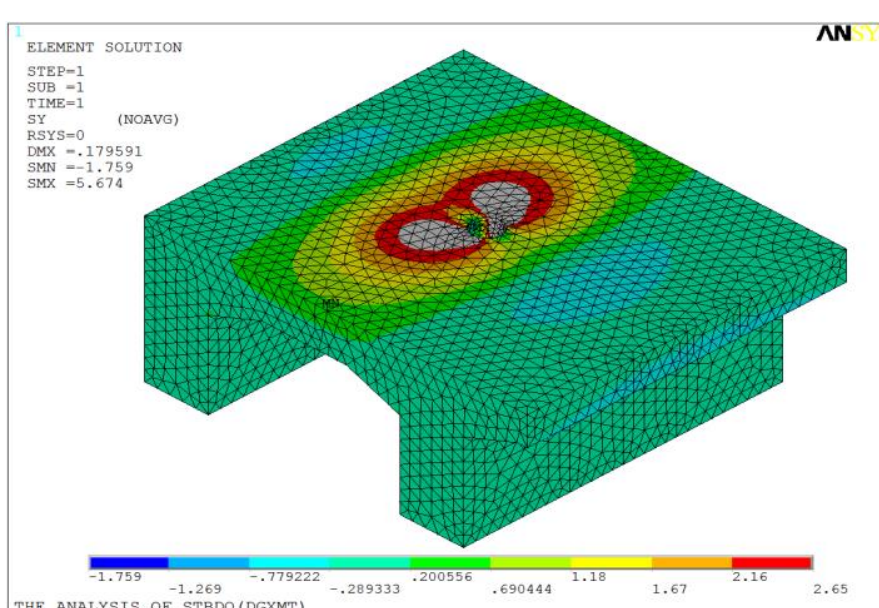

Figure 10. Normal stress of the anchor head top plate under the suspender 
According to the calculation, under the most unfavorable suspender force of the standard composite envelope, the main tensile stress in most areas of the anchor head under the suspender is less than the allowable value of 1.855 in the code. The local stress concentration exists in the concrete position of the anchor head (Figure 6), and the local main tensile stress exceeds the limit at the junction of the anchor block and the web. However, considering the contribution of regular reinforcement, the force meets the specification requirements.

According to the calculation, under the most unfavorable suspender force of the standard combination envelope, except for the stress concentration in a small area, the main compressive stress in most areas is less than the allowable value of $19.44 \mathrm{mpa}$, and the checking calculation of the main compressive stress meets the requirements of the specification (Figure 7).

According to the calculation, under the action of the most unfavorable suspender force in the envelope of the standard combination, the maximum normal stress of the top plate along the bridge in the suspender area is 2.46mpa (Figure 8), which occurs only in a small local range, and is less than the allowable value of 1.83mpa in most other areas (Figure 9). Considering that the ordinary steel bars in the top plate participate in the stress, the stress meets the requirements of the code ${ }^{[3]}$.

According to the calculation, under the action of the most unfavorable suspender force of the standard combination envelope of the standard combination, there is a stress concentration phenomenon in the local area of the horizontal bridge of the top plate in the suspender area, and most other areas are less than the allowable value of $1.83 \mathrm{Mpa}$ in the specification (Figure 10). After considering the stress of the regular reinforcement in the top plate, the stress meets the specification requirements.

\subsection{Local analysis of steel-concrete joint section}

The steel-concrete joint section near the arch foot is selected for local analysis as in Figure 11. The finite element model of the steel-concrete joint section near the arch foot is established by ANSYS. The concrete part is simulated by SOLID65 solid element, the steel structure part is simulated by shell63 shell element, and the plate and shell element, prestressed element and solid element in the steel-concrete joint section are mixed and meshed, which are coupled by constraint process.

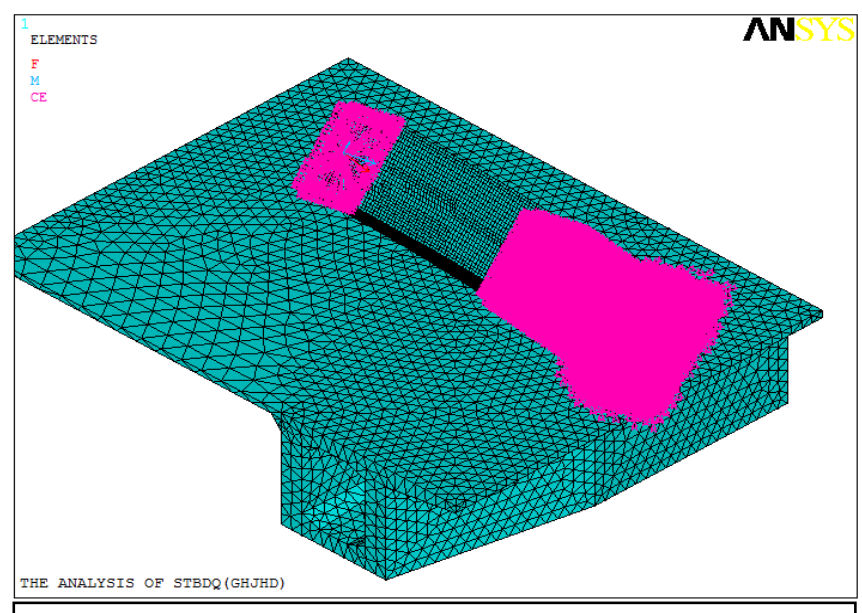

Figure 11. Finite element mesh and constraint equation of steel-concrete section

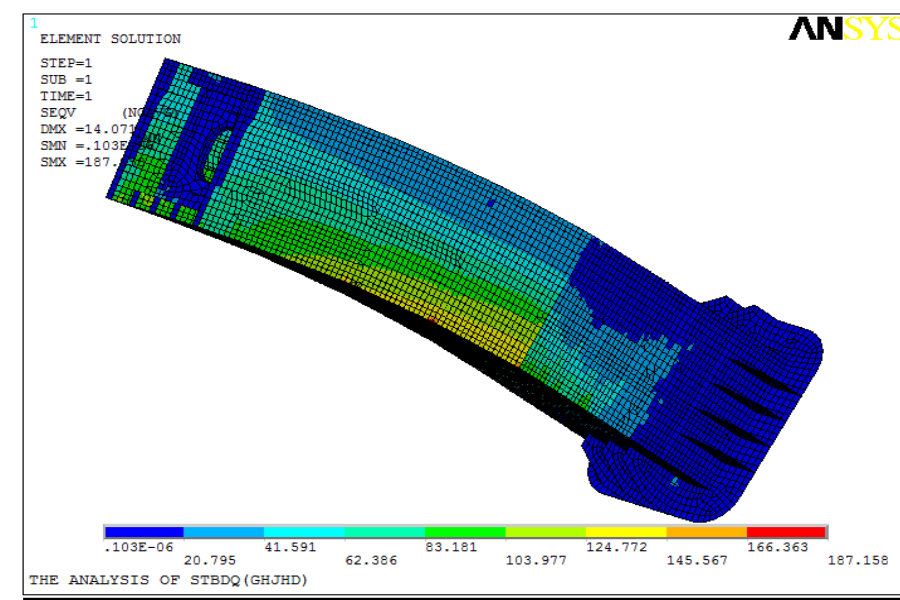

Figure 12. Cloud diagram of equivalent stress of steel structure unit in steel-concrete joint section

Select the most unfavorable load under the standard combination envelope state for loading. As shown in Figure 12, under the standard combination envelope, the maximum equivalent stress of the steel structure unit in the steel-concrete joint section is $187.2 \mathrm{mpa}$, which is less than the yield strength $345 \mathrm{mpa}$. The stress of the steel structure unit in the steel-concrete joint section meets the specification requirements ${ }^{[4]}$. 
Similarly, in the case of standard combined envelope, other components of steel-concrete joint section are as follows:

(1) The maximum equivalent stress of diaphragm unit is $77.8 \mathrm{MPa}$, which is less than the yield strength of $345 \mathrm{MPa}$. The stress of diaphragm unit in steel-concrete joint section meets the specification requirements.

(2) The maximum equivalent stress of the stiffened steel plate in the steel-concrete joint section is $27.7 \mathrm{MPa}$, which is less than the yield strength $345 \mathrm{MPa}$, meeting the specification requirements.

(3) The maximum equivalent stress of the stiffening rib plate unit in is $124.2 \mathrm{MPa}$, which is less than the yield strength $345 \mathrm{MPa}$, meeting the specification requirements.

(4) The maximum equivalent stress of the steel plate unit of the top plate of the arch rib steel box is $114.8 \mathrm{MPa}$, which is less than the yield strength $345 \mathrm{MPa}$, meeting the specification requirements.

(5) The maximum equivalent stress of the steel plate unit of the bottom plate of the arch rib steel box is $187.2 \mathrm{MPa}$, which is less than the yield strength $345 \mathrm{MPa}$, meeting the specification requirements.

(6) The maximum equivalent stress of the steel plate unit of the web of the arch rib steel box is $183.8 \mathrm{MPa}$, which is less than the yield strength $345 \mathrm{MPa}$, meeting the specification requirements.

(7) The maximum equivalent stress of arch box stiffener steel plate unit is $179.1 \mathrm{mpa}$, which is less than the yield strength of $345 \mathrm{mpa}$, meeting the requirements of the specification.

(8) The maximum equivalent stress of the base plate steel plate unit in is $40.3 \mathrm{MPa}$, which is less than the yield strength $345 \mathrm{MPa}$, meeting the specification requirements.

(9) In addition to the distortion of local principal tensile stress in the prestressed anchorage zone, the principal tensile stress in most other areas of the steel box is less than the allowable value of $1.855 \mathrm{MPa}$.

(10) In addition to the distortion of local principal compressive stress in the prestressed anchorage area, the principal compressive stress in most other areas of steel box is less than the allowable value of 19.44mpa.

(11) In addition to extend standard of extremely small areas, the normal tensile stress of the concrete of the main beam outside the box arch along the bridge is lower than the allowable value of $1.83 \mathrm{MPa}$. Considering that the reinforcement is involved in the force, the checking calculation of the normal tensile stress of the main girder along the bridge meets the requirements of the code.

(12) The maximum normal tensile stress of the concrete transverse bridge of the main beam outside the box arch is $1.97 \mathrm{MPa}$, slightly greater than the allowable value of $1.83 \mathrm{MPa}$ in the code. Considering that the reinforcement is involved in the force, the checking calculation of the normal tensile stress of the main girder along the bridge meets the requirements of the code. 


\section{Conclusion}

To sum up, during the design of small and medium span basket type steel box tied arch bridge, the design unit must make local analysis and checking calculation for key parts such as the upper and lower anchor ends of suspenders and steel-concrete joint sections. Only by ensuring the design requirements of key parts can we effectively ensure the quality and safety of the whole project and meet its actual construction and application needs. This has far-reaching significance for the design, construction and application of small and medium span basket type steel box tied arch bridge.

\section{Disclosure statement}

The author declares no conflict of interest.

\section{References}

[1] Liu LL, Yu Q, Qiu Y, Wang D, 2021, Research on Structural Design of Steel-concrete Joint Section of Arch Foot of Steel Box and Rigid Frame Tied Arch Bridge. Technology of Highway and Transport (03): 78-83.

[2] Xu C, 2021, Analysis of the Influence of Transverse Support on the Stability of Steel Box Tied Arch Bridge. Science and Technology Innovation (16): 142-143.

[3] Li JY, 2019, Research on Arch Rib Alignment Control and Derrick Force Optimization of Steel Box Tied Arch Bridge. Tutor: Jiang Nan. Southwest Jiaotong University.

[4] Lu Y, 2021, Stress Analysis of Steel Structure in Suspension Anchorage Area of a Tied Arch Bridge. Shanghai Highways, (02): 54-56. 